UDC: 517.518 .28

45 (LXXI) No.2 (2021), 149-175

DOI: $10.37560 /$ matbil21452151k

\title{
NEW INEQUALITIES FOR APPROXIMATELY $h$-CONVEX FUNCTIONS PERTAINING GENERALIZED FRACTIONAL INTEGRALS
}

\author{
ARTION KASHURI ${ }^{1}$, HÜSEYIN BUDAK ${ }^{2}$, RABIA KAPUCU $^{2}$, AND ROZANA LIKO $^{1}$
}

\begin{abstract}
In this paper, we first establish some new Hermite-Hadamard type inequalities for approximately $h$-convex function by using generalized fractional integrals. Also, some midpoint and trapezoid type inequalities for approximately $h$-convex functions via generalized fractional integrals are presented. At the end, a briefly conclusion is given. Our results may stimulate further research in different areas of pure and applied sciences.
\end{abstract}

\section{Introduction and Preliminaries}

Definition 1.1. A function $f: I \subseteq \Re \rightarrow \Re$ is said to be convex, if

$$
f(t x+(1-t) y) \leq t f(x)+(1-t) f(y)
$$

for all $x, y \in I$ and $t \in[0,1]$.

The inequalities discovered by C. Hermite and J. Hadamard for convex functions are considerable significant in the literature (see, e.g., [6, 12, [28, p. 137]). These inequalities state that, if $f: I \rightarrow \Re$ is a convex function on the interval $I$ of real numbers and $a, b \in I$, with $a<b$, then

$$
f\left(\frac{a+b}{2}\right) \leq \frac{1}{b-a} \int_{a}^{b} f(x) d x \leq \frac{f(a)+f(b)}{2} .
$$

Both inequalities hold in the reversed direction if $f$ is concave. We note that Hadamard's inequality may be regarded as a refinement of the concept of convexity and it follows easily from Jensen's inequality. A number of mathematicians have devoted their efforts to generalise, refine, counterpart and extend it for different classes of functions such as using convex mappings. Sarikaya et al. obtain the Hermite-Hadamard inequality for the Riemann-Liouville fractional integrals

2010 Mathematics Subject Classification. Primary: 26D07 Secondary: 26D10, 26D15, 26B15, 26B25, 26A33.

Key words and phrases. Hermite-Hadamard integral inequality, approximately $h$-convex function, general fractional integrals. 
in [33. Also, many authors have studied to generalize this inequality and establish Hermite-Hadamard inequality for other fractional integrals such as $k$-fractional integral, Hadamard fractional integrals, Katugampola fractional integrals, Conformable fractional integrals, etc. For some of them, please see [2, 5, 9, 10, [13][19], [23, 24, 32, 34], 36]-[40, [42, 43]. For more information about fractional calculus, please refer to [11, 20, 44, 45].

In [7], Dragomir and Agarwal establish the following identity and using it, they present some bounds for the right hand side of the inequality 1.2 .

Lemma 1. Let $f: I^{*} \rightarrow \Re$ be differentiable function on $I^{*}, a, b \in I^{*}$ ( $I^{*}$ is interior of $I)$, with $a<b$. If $f^{\prime} \in L[a, b]$, then the following equality holds:

$$
\frac{f(a)+f(b)}{2}-\frac{1}{b-a} \int_{a}^{b} f(t) d t=\frac{(b-a)}{2} \int_{0}^{1}(1-2 t) f^{\prime}(t a+(1-t) b) d t .
$$

In [21, Kirmaci gives the following identity and using this identity, obtains some bounds for the left hand side of the inequality 1.2

Lemma 2. Let $f: I^{*} \rightarrow \Re$ be differentiable function on $I^{*}, a, b \in I^{*}$ ( $I^{*}$ is interior of I), with $a<b$. If $f^{\prime} \in L[a, b]$, then we have

$$
\begin{gathered}
\frac{1}{b-a} \int_{a}^{b} f(t) d t-f\left(\frac{a+b}{2}\right) \\
=(b-a)\left[\int_{0}^{\frac{1}{2}} t f^{\prime}(t a+(1-t) b) d t+\int_{\frac{1}{2}}^{1}(1-t) f^{\prime}(t a+(1-t) b) d t\right] .
\end{gathered}
$$

Over the last twenty years, the numerous studies have focused on to obtain new bound for left hand side and right and side of the inequality 1.2 . For some examples, please refer to [8, 22, [25]-[27, [29, 30, 35].

\section{2. $h$-CONVEXITY AND NEW GENERALIZED FRACTIONAL INTEGRAL OPERATORS}

First, we give some useful definitions:

Definition 2.1. [1] A non negative function $f:[a, b] \rightarrow \Re$ is said to be $s$-convex in second sense, if

$$
f(t a+(1-t) b) \leq t^{s} f(a)+(1-t)^{s} f(b)
$$

holds for all $t \in[0,1]$ and $s \in(0,1]$.

In 41], Varošanec defined generalization of convex, $s$-convex, Godunova-Levin functions and $P$-convex functions as follows:

Definition 2.2. [41] Let $h: J \rightarrow \Re$ be non negative function and $h \neq 0$. A non negative function $f: I=[a, b] \rightarrow \Re$ is called $h$-convex, if

$$
f(t a+(1-t) b) \leq h(t) f(a)+h(1-t) f(b)
$$

holds for all $t \in[0,1]$. For more discussion about the h-convex function see [41]. We denote by $S X(h, I)$ the class of $h$-convex functions on $I$. 
We let $\left(X,\|\cdot\|_{I}\right)$ be a normed quasi-linear space, $I$ is nonempty convex subset of $X, d: X \times X \rightarrow \Re$.

Definition 2.3. [4 Let $h:[0,1] \rightarrow \Re$ be non negative function and $h \neq 0$. A function $f: I \rightarrow \Re$ is said to be approximately $h$-convex, if

$$
f(t x+(1-t) y) \leq h(t) f(x)+h(1-t) f(y)+d(x, y)
$$

holds for all $t \in(0,1)$ and $a, b \in I$.

Now we summarize the generalized fractional integrals defined by Sarikaya and Ertuğral in [31.

Let's define a function $\varphi:[0,+\infty) \rightarrow[0,+\infty)$ satisfiying the following conditions:

$$
\begin{aligned}
& \int_{0}^{1} \frac{\varphi(t)}{t} d t<+\infty, \\
& \frac{1}{A} \leq \frac{\varphi(s)}{\phi(r)} \leq A \text { for } \frac{1}{2} \leq \frac{s}{r} \leq 2, \\
& \frac{\varphi(r)}{r^{2}} \leq B \frac{\varphi(s)}{s^{2}} \text { for } s \leq r, \\
& \left|\frac{\varphi(r)}{r^{2}}-\frac{\varphi(s)}{s^{2}}\right| \leq C|r-s| \frac{\varphi(r)}{r^{2}} \text { for } \frac{1}{2} \leq \frac{s}{r} \leq 2,
\end{aligned}
$$

where $A, B, C>0$ are independent of $r, s>0$.

We define the following left-sided and right-sided generalized fractional integral operators, respectively, as follows:

$$
\begin{aligned}
& { }_{a^{+}} I_{\varphi} f(x)=\int_{a}^{x} \frac{\varphi(x-t)}{x-t} f(t) d t, \quad x>a, \\
& { }_{b^{-}} I_{\varphi} f(x)=\int_{x}^{b} \frac{\varphi(t-x)}{t-x} f(t) d t, \quad x<b .
\end{aligned}
$$

The most important feature of generalized fractional integrals is that they generalize some types of fractional integrals such as Riemann-Liouville fractional integral, $k$-Riemann-Liouville fractional integral, Katugampola fractional integrals, conformable fractional integral, Hadamard fractional integrals, etc.

Sarıkaya and Ertuğral also establish the following Hermite-Hadamard inequality for the generalized fractional integral operators:

Theorem 1. [31] Let $f:[a, b] \rightarrow \Re$ be a convex function on $[a, b]$, with $a<b$, then the following inequalities for fractional integral operators hold:

$$
f\left(\frac{a+b}{2}\right) \leq \frac{1}{2 \Lambda(1)}\left[a+I_{\varphi} f(b)+{ }_{b-} I_{\varphi} f(a)\right] \leq \frac{f(a)+f(b)}{2},
$$

where the mapping $\Lambda:[0,1] \rightarrow \Re$ is defined by

$$
\Lambda(x):=\int_{0}^{x} \frac{\varphi((b-a) t)}{t} d t .
$$


Budak et al. proved the following another version of Hermite-Hadamard inequality for the generalized fractional integral operators:

Theorem 2. [3] Let $f:[a, b] \rightarrow \Re$ be a function, with $a<b$, and $f \in L_{1}[a, b]$. If $f$ is a convex function on $[a, b]$, then we have the following inequalities for generalized fractional integral operators:

$$
f\left(\frac{a+b}{2}\right) \leq \frac{1}{2 \Psi(1)}\left[\left(\frac{a+b}{2}\right)+I_{\varphi} f(b)+\left(\frac{a+b}{2}\right)-I_{\varphi} f(a)\right] \leq \frac{f(a)+f(b)}{2},
$$

where the mapping $\Psi:[0,1] \rightarrow \Re$ is defined by

$$
\Psi(x):=\int_{0}^{x} \frac{\varphi\left(\frac{(b-a)}{2} t\right)}{t} d t .
$$

Motivated by the above literature, the main objective of this article is to establish in section 3 new Hermite-Hadamard type inequalities for approximately $h$-convex function by using generalized fractional integrals given in section 2, Also, some midpoint and trapezoid type inequalities for approximately $h$-convex functions via generalized fractional integrals are presented, respectively. Moreover a big remark is given for the special cases of the functions $h$ and $\varphi$. In section 4 a briefly conclusion is given as well.

\section{Hermite-Hadamard type inequalities FOR APPROXIMATELY $h$-CONVEX FUNCTIONS}

In this section, we establish Hermite-Hadamard inequalities for approximately $h-$ convex functions via generalized fractional integrals summarized above.

Theorem 3. Let $f:[a, b] \rightarrow \Re$ be an approximately $h$-convex on $[a, b]$, with $a<b$. Then the following inequalities for generalized fractional integrals hold:

$$
\begin{aligned}
\frac{1}{2 h\left(\frac{1}{2}\right)} f\left(\frac{a+b}{2}\right)-A_{1} \leq & \frac{1}{2 \Lambda(1)}\left[a+I_{\varphi} f(b)+{ }_{b-} I_{\varphi} f(a)\right] \\
\leq & \frac{[f(a)+f(b)]}{2 \Lambda(1)} \int_{0}^{1} \frac{\varphi((b-a) t)}{t}[h(t)+h(1-t)] d t \\
& +d(a, b),
\end{aligned}
$$

where

$$
A_{1}:=\frac{1}{2 \Lambda(1) h\left(\frac{1}{2}\right)} \int_{a}^{b} \frac{\varphi(b-x)}{b-x} d(x, a+b-x) d x .
$$

Proof. Since $f$ is an approximately $h$-convex, we have the following inequality

$$
f\left(\frac{x+y}{2}\right) \leq h\left(\frac{1}{2}\right)[f(x)+f(y)]+d(x, y) .
$$


For $x=t a+(1-t) b$ and $y=t b+(1-t) a$ with $t \in[0,1], 3.2$ becomes

$$
\begin{aligned}
\frac{1}{h\left(\frac{1}{2}\right)} f\left(\frac{a+b}{2}\right) \leq & f(t a+(1-t) b)+f(t b+(1-t) a) \\
& +\frac{1}{h\left(\frac{1}{2}\right)} d(t a+(1-t) b, t b+(1-t) a) .
\end{aligned}
$$

Multiplying both sides of the inequality $\sqrt{3.3}$ by $\frac{\varphi((b-a) t)}{t}$ and integrating the resultant inequality with respect to $t$ over $[0,1]$, we obtain

$$
\begin{aligned}
& \frac{1}{h\left(\frac{1}{2}\right)} f\left(\frac{a+b}{2}\right) \int_{0}^{1} \frac{\varphi((b-a) t)}{t} d t \\
\leq & \int_{0}^{1} \frac{\varphi((b-a) t)}{t} f(t a+(1-t) b) d t+\int_{0}^{1} \frac{\varphi((b-a) t)}{t} f(t b+(1-t) a) d t \\
& +\frac{1}{h\left(\frac{1}{2}\right)} \int_{0}^{1} \frac{\varphi((b-a) t)}{t} d(t a+(1-t) b, t b+(1-t) a) d t .
\end{aligned}
$$

As a consequence, we get

$$
\begin{aligned}
\frac{1}{2 h\left(\frac{1}{2}\right)} f\left(\frac{a+b}{2}\right) \leq & \frac{1}{2 \Lambda(1)}\left[a+I_{\varphi} f(b)+{ }_{b-} I_{\varphi} f(a)\right] \\
& +\frac{1}{2 \Lambda(1) h\left(\frac{1}{2}\right)} \int_{a}^{b} \frac{\varphi(b-x)}{b-x} d(x, a+b-x) d x
\end{aligned}
$$

and thus the first inequality is proved.

To obtain the second inequality in $(3.1)$, since $f$ is an approximately $h$-convex, we have

$$
f(t a+(1-t) b)+f(t b+(1-t) a) \leq[f(a)+f(b)][h(t)+h(1-t)]+2 d(a, b) .
$$

Then multiplying both sides of 3.4 by $\frac{\varphi((b-a) t)}{t}$ and integrating the resultant inequality with respect to $t$ on $[0,1]$, we have

$$
\begin{aligned}
& \int_{0}^{1} \frac{\varphi((b-a) t)}{t} f(t a+(1-t) b) d t \\
& +\int_{0}^{1} \frac{\varphi((b-a) t)}{t} f(t b+(1-t) a) d t+2 d(a, b) \int_{0}^{1} \frac{\varphi((b-a) t)}{t} d t \\
& \leq \quad[f(a)+f(b)] \int_{0}^{1} \frac{\varphi((b-a) t)}{t}[h(t)+h(1-t)] d t+2 d(a, b) \Lambda(1) .
\end{aligned}
$$


As a sequel, we get

$$
\begin{aligned}
& \frac{1}{2 \Lambda(1)}\left[{ }_{a+} I_{\varphi} f(b)+{ }_{b-} I_{\varphi} f(a)\right] \\
\leq & \frac{[f(a)+f(b)]}{2 \Lambda(1)} \int_{0}^{1} \frac{\varphi((b-a) t)}{t}[h(t)+h(1-t)] d t+d(a, b)
\end{aligned}
$$

and the second inequality is proved. The proof is completed.

Theorem 4. Let $f, g:[a, b] \rightarrow \Re$ are two approximately $h$-convex functions on $[a, b]$, with $a<b$. Then the following inequality for generalized fractional integrals holds:

$$
\begin{aligned}
& \frac{1}{2}\left[{ }_{a+} I_{\varphi} f(b) g(b)+{ }_{b-} I_{\varphi} f(a) g(a)\right] \\
\leq & K_{1} M(a, b)+K_{2} N(a, b) d(a, b)+K_{3} P(a, b) d(a, b),
\end{aligned}
$$

where

$$
\begin{gathered}
M(a, b):=f(a) g(a)+f(b) g(b), \quad N(a, b):=f(a) g(b)+f(b) g(a), \\
P(a, b):=f(a)+g(a)+f(b)+g(b),
\end{gathered}
$$

and

$$
\begin{aligned}
K_{1} & :=\frac{1}{2} \int_{0}^{1} \frac{\varphi((b-a) t)}{t}\left[h^{2}(t)+h^{2}(1-t)\right] d t, \\
K_{2} & :=\int_{0}^{1} \frac{\varphi((b-a) t)}{t} h(t) h(1-t) d t, \\
K_{3} & :=\frac{1}{2} \int_{0}^{1} \frac{\varphi((b-a) t)}{t}[h(t)+h(1-t)] d t .
\end{aligned}
$$

Proof. Since $f, g$ are approximately $h$-convex functions for $t \in[0,1]$, we have

$$
f(t a+(1-t) b) \leq h(t) f(a)+h(1-t) f(b)+d(a, b)
$$

and

$$
g(t a+(1-t) b) \leq h(t) g(a)+h(1-t) g(b)+d(a, b) .
$$

Multiplying (3.7) and (3.8), we get

$$
\begin{aligned}
& f(t a+(1-t) b) g(t a+(1-t) b) \\
\leq & h^{2}(t) f(a) g(a)+h^{2}(1-t) f(b) g(b)+h(t) h(1-t)[f(a) g(b)+f(b) g(a)]
\end{aligned}
$$




$$
+h(t) d(a, b)[f(a)+g(a)]+h(1-t) d(a, b)[f(b)+g(b)]+d^{2}(a, b) .
$$

Similarly, we obtain

$$
\begin{aligned}
& f(t b+(1-t) a) g(t b+(1-t) a) \\
\leq & h^{2}(1-t) f(a) g(a)+h^{2}(t) f(b) g(b)+h(t) h(1-t)[f(a) g(b)+f(b) g(a)] \\
& +h(t) d(a, b)[f(b)+g(b)]+h(1-t) d(a, b)[f(a)+g(a)]+d^{2}(a, b) .
\end{aligned}
$$

Adding (3.9) and $(3.10)$, we have the following relation

$$
\begin{aligned}
& f(t a+(1-t) b) g(t a+(1-t) b) \\
+ & f(t b+(1-t) a) g(t b+(1-t) a) \\
\leq & {\left[h^{2}(t)+h^{2}(1-t)\right] M(a, b)+2 h(t) h(1-t) N(a, b) } \\
& +[h(t)+h(1-t)] P(a, b) d(a, b)+2 d^{2}(a, b) .
\end{aligned}
$$

Multiplying both sides of 3.11 by $\frac{\varphi((b-a) t)}{t}$ and integrating the resultant one with respect to $t$ over $[0,1]$, we get

$$
\begin{aligned}
& \int_{0}^{1} \frac{\varphi((b-a) t)}{t} f(t a+(1-t) b) g(t a+(1-t) b) d t \\
& +\int_{0}^{1} \frac{\varphi((b-a) t)}{t} f((1-t) a+t b) g((1-t) a+t b) d t \\
& \leq \quad M(a, b) \int_{0}^{1} \frac{\varphi((b-a) t)}{t}\left[h^{2}(t)+h^{2}(1-t)\right] d t \\
& +2 N(a, b) \int_{0}^{1} \frac{\varphi((b-a) t)}{t} h(t) h(1-t) d t \\
& +P(a, b) d(a, b) \int_{0}^{1} \frac{\varphi((b-a) t)}{t}[h(t)+h(1-t)] d t+ \\
& +2 d^{2}(a, b) \int_{0}^{1} \frac{\varphi((b-a) t)}{t} d t .
\end{aligned}
$$


This completes the proof.

Theorem 5. Let $f, g:[a, b] \rightarrow \Re$ are two approximately $h$-convex functions on $[a, b]$, with $a<b$. Then the following inequality for generalized fractional integrals holds:

$$
\begin{aligned}
& \quad \frac{1}{2 h^{2}\left(\frac{1}{2}\right)} f\left(\frac{a+b}{2}\right) g\left(\frac{a+b}{2}\right) \\
& -\frac{1}{2 \Lambda(1) h\left(\frac{1}{2}\right)}\left[\int_{a}^{b} \frac{\varphi(b-x)}{b-x}(f(x)+g(x)) d(x, a+b-x) d x\right. \\
& \left.\quad+\int_{a}^{b} \frac{\varphi(x-a)}{x-a}(f(x)+g(x)) d(a+b-x, x) d x\right] \\
& \leq \quad \frac{1}{2 \Lambda(1)}\left[{ }_{a+} I_{\varphi} f(x) g(x)+{ }_{b-} I_{\varphi} f(x) g(x)\right] \\
& \quad+\frac{1}{2 \Lambda(1) h^{2}\left(\frac{1}{2}\right)} \int_{a}^{b} \frac{\varphi(b-x)}{b-x} d^{2}(x, a+b-x) d x \\
& +\frac{1}{\Lambda(1)}\left[K_{2} M(a, b)+K_{1} N(a, b)+K_{3} P(a, b) d(a, b)\right]+d^{2}(a, b),
\end{aligned}
$$

where $M(a, b), N(a, b)$ and $K_{1}, K_{2}, K_{3}, P(a, b)$ are defined as in Theorem 4 .

Proof. For $t \in[0,1]$, we can write

$$
\frac{a+b}{2}=\frac{(1-t) a+t b}{2}+\frac{t a+(1-t) b}{2} .
$$

Since $f$ and $g$ are two approximately $h$-convex functions, we have

$$
\begin{aligned}
\frac{1}{h\left(\frac{1}{2}\right)} f\left(\frac{a+b}{2}\right)= & \frac{1}{h\left(\frac{1}{2}\right)} f\left(\frac{(1-t) a+t b}{2}+\frac{t a+(1-t) b}{2}\right) \\
\leq & f((1-t) a+t b)+f(t a+(1-t) b) \\
& +\frac{1}{h\left(\frac{1}{2}\right)} d((1-t) a+t b, t a+(1-t) b)
\end{aligned}
$$

and

$$
\begin{aligned}
\frac{1}{h\left(\frac{1}{2}\right)} g\left(\frac{a+b}{2}\right)= & \frac{1}{h\left(\frac{1}{2}\right)} g\left(\frac{(1-t) a+t b}{2}+\frac{t a+(1-t) b}{2}\right) \\
\leq & g((1-t) a+t b)+g(t a+(1-t) b) \\
& +\frac{1}{h\left(\frac{1}{2}\right)} d((1-t) a+t b, t a+(1-t) b) .
\end{aligned}
$$


Multiplying the inequalities $(3.13)$ and $(3.14)$, we obtain

$$
\begin{aligned}
& \frac{1}{h^{2}\left(\frac{1}{2}\right)} f\left(\frac{a+b}{2}\right) g\left(\frac{a+b}{2}\right) \\
\leq & {\left.[f(1-t) a+t b)+f(t a+(1-t) b)+\frac{1}{h\left(\frac{1}{2}\right)} d((1-t) a+t b, t a+(1-t) b)\right] } \\
& \times\left[g((1-t) a+t b)+g(t a+(1-t) b)+\frac{1}{h\left(\frac{1}{2}\right)} d((1-t) a+t b, t a+(1-t) b)\right] \\
= & {[f((1-t) a+t b) g((1-t) a+t b)+f(t a+(1-t) b) g(t a+(1-t) b)] } \\
& +[f((1-t) a+t b) g(t a+(1-t) b)+f(t a+(1-t) b) g((1-t) a+t b)] \\
& +\frac{1}{h\left(\frac{1}{2}\right)}[f(t a+(1-t) b) d(t a+(1-t) b, t b+(1-t) a) \\
& +f(t b+(1-t) a) d(t a+(1-t) b, t b+(1-t) a) \\
& +g(t a+(1-t) b) d(t a+(1-t) b, t b+(1-t) a) \\
+ & +\frac{1}{h^{2}\left(\frac{1}{2}\right)}\left[d^{2}(t a+(1-t) b, t b+(1-t) a)\right]
\end{aligned}
$$

By using the approximately $h$-convexity of functions $f$ and $g$, we get

$$
\begin{aligned}
& \frac{1}{h^{2}\left(\frac{1}{2}\right)} f\left(\frac{a+b}{2}\right) g\left(\frac{a+b}{2}\right) \\
\leq \quad & {[f((1-t) a+t b) g((1-t) a+t b)+f(t a+(1-t) b) g(t a+(1-t) b)] } \\
& +\left[h^{2}(t)+h^{2}(1-t)\right] N(a, b)+2 h(t) h(1-t) M(a, b) \\
& +[h(t)+h(1-t)] P(a, b) d(a, b)+2 d^{2}(a, b) \\
& +\frac{1}{h\left(\frac{1}{2}\right)}[f(t a+(1-t) b) d(t a+(1-t) b, t b+(1-t) a)] \\
& +\frac{1}{h\left(\frac{1}{2}\right)}[f(t b+(1-t) a) d(t a+(1-t) b, t b+(1-t) a)] \\
+ & \frac{1}{h\left(\frac{1}{2}\right)} g[(t a+(1-t) b) d(t a+(1-t) b, t b+(1-t) a)]
\end{aligned}
$$




$$
\begin{aligned}
& +\frac{1}{h\left(\frac{1}{2}\right)} g[(t b+(1-t) a) d(t a+(1-t) b, t b+(1-t) a)] \\
& +\frac{1}{h^{2}\left(\frac{1}{2}\right)} d^{2}(t a+(1-t) b, t b+(1-t) a) .
\end{aligned}
$$

Multiplying both sides of inequality 3.15 by $\frac{\varphi((b-a) t)}{t}$ and integrating the resultant one with respect to $t$ over $[0,1]$, we obtain

$$
\begin{aligned}
& \frac{1}{h^{2}\left(\frac{1}{2}\right)} \int_{0}^{1} \frac{\varphi((b-a) t)}{t} f\left(\frac{a+b}{2}\right) g\left(\frac{a+b}{2}\right) d t \\
\leq & \int_{0}^{1} \frac{\varphi((b-a) t)}{t} f((1-t) a+t b) g((1-t) a+t b) d t \\
& +\int_{0}^{1} \frac{\varphi((b-a) t)}{t} f(t a+(1-t) b) g(t a+(1-t) b) d t \\
& +\frac{1}{h^{2}\left(\frac{1}{2}\right)} \int_{0}^{1} \frac{\varphi((b-a) t)}{t} d^{2}(t a+(1-t) b, t b+(1-t) a) d t \\
& +\frac{1}{h\left(\frac{1}{2}\right)} \int_{0}^{1} \frac{\varphi((b-a) t)}{t} f(t a+(1-t) b) d(t a+(1-t) b, t b+(1-t) a) d t \\
& +\frac{1}{h\left(\frac{1}{2}\right)} \int_{0}^{1} \frac{\varphi((b-a) t)}{t} f(t b+(1-t) a) d(t a+(1-t) b, t b+(1-t) a) d t \\
& +\frac{1}{h\left(\frac{1}{2}\right)} \int_{0}^{1} \frac{\varphi((b-a) t)}{t} g(t a+(1-t) b) d(t a+(1-t) b, t b+(1-t) a) d t \\
& +\frac{1}{h\left(\frac{1}{2}\right)} \int_{0}^{1} \frac{\varphi((b-a) t)}{t} g(t b+(1-t) a) d(t a+(1-t) b, t b+(1-t) a) d t \\
& +2 N(a, b) \int_{0}^{1} \frac{\varphi((b-a) t)}{t} h^{2}(t) d t \\
+ & 2 M(a, b) \int_{0}^{1} \frac{\varphi((b-a) t)}{t} h(t) h(1-t) d t \\
+ & 2 P(a, b) d(a, b) \int_{0}^{1} \frac{\varphi((b-a) t)}{t}[h(t)+h(1-t)] d t \\
+ & 2 d^{2}(a, b) \int_{0}^{1} \frac{\varphi((b-a) t)}{t} d t .
\end{aligned}
$$

By changing the variable of integration we achieved desired inequality (3.12).

Theorem 6. Let $f:[a, b] \rightarrow \Re$ be an approximately $h$-convex function on $[a, b]$, with $a<b$. Then the following inequalities for generalized fractional integrals hold:

$$
\begin{aligned}
& \frac{1}{2 h\left(\frac{1}{2}\right)} f\left(\frac{a+b}{2}\right)-A_{2} \\
\leq & \frac{1}{2 \Psi(1)}\left[\left(\frac{a+b}{2}\right)+I_{\varphi} f(b)+\left(\frac{a+b}{2}\right)-I_{\varphi} f(a)\right]
\end{aligned}
$$




$$
\leq \frac{[f(a)+f(b)]}{2 \Psi(1)} \int_{0}^{1} \frac{\varphi\left(\left(\frac{b-a}{2}\right) t\right)}{t}\left[h\left(\frac{t}{2}\right)+h\left(\frac{2-t}{2}\right)\right] d t+d(a, b),
$$

where

$$
A_{2}:=\frac{1}{2 \Psi(1) h\left(\frac{1}{2}\right)} \int_{\frac{a+b}{2}}^{b} \frac{\varphi(b-x)}{b-x} d(x, a+b-x) d x .
$$

Proof. Since $f$ is an approximately $h$-convex, we have the following inequality

$$
f\left(\frac{x+y}{2}\right) \leq h\left(\frac{1}{2}\right)[f(x)+f(y)]+d(x, y) .
$$

For $x=\frac{t}{2} a+\frac{(2-t)}{2} b$ and $y=\frac{t}{2} b+\frac{(2-t)}{2} a$ with $t \in[0,1], 3.17$ becomes

$$
\begin{aligned}
& \frac{1}{h\left(\frac{1}{2}\right)} f\left(\frac{a+b}{2}\right) \\
\leq & f\left(\frac{t}{2} a+\frac{(2-t)}{2} b\right)+f\left(\frac{t}{2} b+\frac{(2-t)}{2} a\right) \\
+ & \frac{1}{h\left(\frac{1}{2}\right)} d\left(\frac{t}{2} a+\frac{(2-t)}{2} b, \frac{t}{2} b+\frac{(2-t)}{2} a\right) .
\end{aligned}
$$

Multiplying both sides of the inequality 3.18 by $\frac{\varphi\left(\frac{(b-a)}{2} t\right)}{t}$ and integrating the resultant inequality with respect to $t$ over $[0,1]$, we have

$$
\begin{aligned}
& \frac{1}{h\left(\frac{1}{2}\right)} f\left(\frac{a+b}{2}\right) \int_{0}^{1} \frac{\varphi\left(\left(\frac{b-a}{2}\right) t\right)}{t} d t \\
\leq & \int_{0}^{1} \frac{\varphi\left(\left(\frac{b-a}{2}\right) t\right)}{t} f\left(\frac{t}{2} a+\frac{(2-t)}{2} b\right) d t+\int_{0}^{1} \frac{\varphi\left(\left(\frac{b-a}{2}\right) t\right)}{t}\left(\frac{t}{2} b+\frac{(2-t)}{2} a\right) d t \\
& +\frac{1}{h\left(\frac{1}{2}\right)} \int_{0}^{1} \frac{\varphi\left(\left(\frac{b-a}{2}\right) t\right)}{t} d\left(\frac{t}{2} a+\frac{(2-t)}{2} b, \frac{t}{2} b+\frac{(2-t)}{2} a\right) d t .
\end{aligned}
$$

As a consequence, we get

$$
\begin{aligned}
\frac{1}{2 h\left(\frac{1}{2}\right)} f\left(\frac{a+b}{2}\right) \leq & \frac{1}{2 \Psi(1)}\left[\left(\frac{a+b}{2}\right)+I_{\varphi} f(b)+\left(\frac{a+b}{2}\right)-I_{\varphi} f(a)\right] \\
& +\frac{1}{2 \Psi(1) h\left(\frac{1}{2}\right)} \int_{\frac{a+b}{2}}^{b} \frac{\varphi(b-x)}{b-x} d(x . a+b-x) d x
\end{aligned}
$$

and thus the first inequality is proved. 
To obtain the second inequality in $(3.16)$, since $f$ is an approximately $h$-convex, we have

$$
\begin{aligned}
& f\left(\frac{t}{2} a+\frac{(2-t)}{2} b\right)+f\left(\frac{t}{2} b+\frac{(2-t)}{2} a\right) \\
\leq & {[f(a)+f(b)]\left[h\left(\frac{t}{2}\right)+h\left(\frac{2-t}{2}\right)\right]+2 d(a, b) . }
\end{aligned}
$$

Then multiplying both sides of 3.19 by $\frac{\varphi\left(\left(\frac{(b-a)}{2}\right) t\right)}{t}$ and integrating the resultant inequality with respect to $t$ on $[0,1]$, we get

$$
\begin{aligned}
& \int_{0}^{1} \frac{\varphi\left(\left(\frac{b-a}{2}\right) t\right)}{t} f\left(\frac{t}{2} a+\frac{(2-t)}{2} b\right) d t \\
& +\int_{0}^{1} \frac{\varphi\left(\left(\frac{b-a}{2}\right) t\right)}{t} f\left(\frac{t}{2} b+\frac{(2-t)}{2} a\right) d t+2 d(a, b) \int_{0}^{1} \frac{\varphi\left(\left(\frac{b-a}{2}\right) t\right)}{t} d t \\
& \leq \quad[f(a)+f(b)] \int_{0}^{1} \frac{\varphi\left(\left(\frac{b-a}{2}\right) t\right)}{t}\left[h\left(\frac{t}{2}\right)+h\left(\frac{2-t}{2}\right)\right] d t+2 d(a, b) \Psi(1) .
\end{aligned}
$$

As a sequel, we obtain

$$
\begin{aligned}
& \frac{1}{2 \Psi(1)}\left[\left(\frac{a+b}{2}\right)+I_{\varphi} f(b)+\left(\frac{a+b}{2}\right)-I_{\varphi} f(a)\right] \\
\leq & \frac{[f(a)+f(b)]}{2 \Psi(1)} \int_{0}^{1} \frac{\varphi\left(\left(\frac{b-a}{2}\right) t\right)}{t}\left[h\left(\frac{t}{2}\right)+h\left(\frac{2-t}{2}\right)\right] d t+d(a, b)
\end{aligned}
$$

and the second inequality is proved. The proof is completed.

Theorem 7. Let $f, g:[a, b] \rightarrow \Re$ are two approximately $h$-convex functions on $[a, b]$, with $a<b$. Then the following inequality for generalized fractional integrals holds:

$$
\begin{aligned}
& \frac{1}{2}\left[\left(\frac{a+b}{2}\right)+I_{\varphi} f(b) g(b)+\left(\frac{a+b}{2}\right)-I_{\varphi} f(a) g(a)\right] \\
\leq & K_{4} M(a, b)+K_{5} N(a, b)+K_{6} P(a, b) d(a, b)+d^{2}(a, b) \Psi(1),
\end{aligned}
$$

where $M(a, b), N(a, b)$, and $P(a, b)$ are defined as in Theorem 4 and

$$
\begin{aligned}
K_{4} & :=\frac{1}{2} \int_{0}^{1} \frac{\varphi\left(\frac{(b-a)}{2} t\right)}{t}\left[h^{2}\left(\frac{t}{2}\right)+h^{2}\left(\frac{2-t}{2}\right)\right] d t, \\
K_{5} & :=\int_{0}^{1} \frac{\varphi\left(\frac{(b-a)}{2} t\right)}{t} h\left(\frac{t}{2}\right) h\left(\frac{2-t}{2}\right) d t
\end{aligned}
$$




$$
K_{6}:=\frac{1}{2} \int_{0}^{1} \frac{\varphi\left(\frac{(b-a)}{2} t\right)}{t}\left[h\left(\frac{t}{2}\right)+h\left(\frac{2-t}{2}\right)\right] d t .
$$

Proof. Since $f$ and $g$ are approximately $h$-convex functions for $t \in[0,1]$, we have

$$
f\left(\frac{t a+(2-t) b}{2}\right) \leq h\left(\frac{t}{2}\right) f(a)+h\left(\frac{2-t}{2}\right) f(b)+d(a, b)
$$

and

$$
g\left(\frac{t a+(2-t) b}{2}\right) \leq h\left(\frac{t}{2}\right) g(a)+h\left(\frac{2-t}{2}\right) g(b)+d(a, b) .
$$

Multiplying (3.20) and (3.21), we get

$$
\begin{aligned}
& f\left(\frac{t a+(2-t) b}{2}\right) g\left(\frac{t a+(2-t) b}{2}\right) \\
\leq & h^{2}\left(\frac{t}{2}\right) f(a) g(a)+h^{2}\left(\frac{2-t}{2}\right) f(b) g(b)+ \\
& +h\left(\frac{t}{2}\right) h\left(\frac{2-t}{2}\right)[f(a) g(b)+f(b) g(a)]+ \\
& +h\left(\frac{t}{2}\right) d(a, b)[f(a)+g(a)]+h\left(\frac{2-t}{2}\right) d(a, b)[f(b)+g(b)]+d^{2}(a, b) .
\end{aligned}
$$

Similarly, we obtain

$$
\begin{aligned}
& f\left(\frac{t b+(2-t) a}{2}\right) g\left(\frac{t b+(2-t) a}{2}\right) \\
\leq & h^{2}\left(\frac{t}{2}\right) f(b) g(b)+h^{2}\left(\frac{2-t}{2}\right) f(a) g(a)+ \\
& +h\left(\frac{t}{2}\right) h\left(\frac{2-t}{2}\right)[f(a) g(b)+f(b) g(a)]+ \\
& +h\left(\frac{t}{2}\right) d(a, b)[f(b)+g(b)]+h\left(\frac{2-t}{2}\right) d(a, b)[f(a)+g(a)]+d^{2}(a, b)
\end{aligned}
$$

Adding (3.22) and (3.23), we have the following relation

$$
\begin{aligned}
& f\left(\frac{t a+(2-t) b}{2}\right) g\left(\frac{t a+(2-t) b}{2}\right) \\
+ & f\left(\frac{t b+(2-t) a}{2}\right) g\left(\frac{t b+(2-t) a}{2}\right) \\
\leq & {\left[h^{2}\left(\frac{t}{2}\right)+h^{2}\left(\frac{2-t}{2}\right)\right] M(a, b)+2 h\left(\frac{t}{2}\right) h\left(\frac{2-t}{2}\right) N(a, b) }
\end{aligned}
$$


162

$$
+\left[h\left(\frac{t}{2}\right)+h\left(\frac{2-t}{2}\right)\right] P(a, b) d(a, b)+2 d^{2}(a, b) .
$$

Multiplying both sides of 3.24 by $\frac{\varphi\left(\frac{(b-a)}{2} t\right)}{t}$ and integrating the resultant one with respect to $t$ over $[0,1]$, we get

$$
\begin{aligned}
& \int_{0}^{1} \frac{\varphi\left(\frac{b-a}{2} t\right)}{t} f\left(\frac{t a+(2-t) b}{2}\right) g\left(\frac{t a+(2-t) b}{2}\right) d t \\
& +\int_{0}^{1} \frac{\varphi\left(\frac{b-a}{2} t\right)}{t} f\left(\frac{t b+(2-t) a}{2}\right) g\left(\frac{t b+(2-t) a}{2}\right) d t \\
& \leq \quad M(a, b) \int_{0}^{1} \frac{\varphi\left(\frac{b-a}{2} t\right)}{t}\left[h^{2}\left(\frac{t}{2}\right)+h^{2}\left(\frac{2-t}{2}\right)\right] d t \\
& +2 N(a, b) \int_{0}^{1} \frac{\varphi\left(\frac{b-a}{2} t\right)}{t} h\left(\frac{t}{2}\right) h\left(\frac{2-t}{2}\right) d t \\
& +P(a, b) d(a, b) \int_{0}^{1} \frac{\varphi\left(\frac{b-a}{2} t\right)}{t}\left[h\left(\frac{t}{2}\right)+h\left(\frac{2-t}{2}\right)\right] d t \\
& +2 d^{2}(a, b) \int_{0}^{1} \frac{\varphi\left(\frac{b-a}{2} t\right)}{t} d t .
\end{aligned}
$$

This completes the proof.

Theorem 8. Let $f, g:[a, b] \rightarrow \Re$ are two approximately $h$-convex functions on $[a, b]$, with $a<b$. Then the following inequality for generalized fractional integrals holds:

$$
\begin{aligned}
& \quad \frac{1}{2 h^{2}\left(\frac{1}{2}\right)} f\left(\frac{a+b}{2}\right) g\left(\frac{a+b}{2}\right) \\
& -\frac{1}{2 \Psi(1) h\left(\frac{1}{2}\right)}\left[\int_{a}^{\frac{a+b}{2}} \frac{\varphi(x-a)}{x-a}[f(x)+g(x)] d(x, a+b-x) d x\right. \\
& \left.\quad+\int_{\frac{a+b}{2}}^{b} \frac{\varphi(b-x)}{b-x}[f(x)+g(x)] d(a+b-x, x) d x\right] \\
& \leq \frac{1}{2}\left[\left(\frac{a+b}{2}\right)-I_{\varphi} f(a) g(a)+\left(\frac{a+b}{2}\right)+I_{\varphi} f(b) g(b)\right]
\end{aligned}
$$




$$
\begin{aligned}
& +\frac{1}{2 \Psi(1) h^{2}\left(\frac{1}{2}\right)} \int_{a}^{\frac{a+b}{2}} \frac{\varphi(x-a)}{x-a} d(x, a+b-x) d x \\
& +\frac{1}{\Psi(1)}\left[K_{5} M(a, b)+K_{4} N(a, b)+K_{6} P(a, b) d(a, b)\right]+d^{2}(a, b),
\end{aligned}
$$

where $M(a, b), N(a, b), P(a, b)$ and $K_{4}, K_{5}, K_{6}$ are defined as in Theorems 4 and 7 , respectively.

Proof. For $t \in[0,1]$, we can write

$$
\frac{a+b}{2}=\frac{(2-t) a+t b}{4}+\frac{t a+(2-t) b}{4}
$$

Since $f$ and $g$ are two approximately $h$-convex functions, we have

$$
\begin{aligned}
\frac{1}{h\left(\frac{1}{2}\right)} f\left(\frac{a+b}{2}\right)= & \frac{1}{h\left(\frac{1}{2}\right)} f\left(\frac{(2-t) a+t b}{4}+\frac{t a+(2-t) b}{4}\right) \\
\leq & f\left(\frac{(2-t) a+t b}{4}\right)+f\left(\frac{t a+(2-t) b}{4}\right) \\
& +\frac{1}{h\left(\frac{1}{2}\right)} d\left(\frac{(2-t) a+t b}{4}, \frac{t a+(2-t) b}{4}\right)
\end{aligned}
$$

and similarly

$$
\begin{aligned}
\frac{1}{h\left(\frac{1}{2}\right)} g\left(\frac{a+b}{2}\right) \leq & g\left(\frac{(2-t) a+t b}{4}\right)+g\left(\frac{t a+(2-t) b}{4}\right) \\
& +\frac{1}{h\left(\frac{1}{2}\right)} d\left(\frac{(2-t) a+t b}{4}, \frac{t a+(2-t) b}{4}\right) .
\end{aligned}
$$

Multiplying the inequalities $(3.26)$ and 3.27 , we obtain

$$
\begin{aligned}
& \frac{1}{h^{2}\left(\frac{1}{2}\right)} f\left(\frac{a+b}{2}\right) g\left(\frac{a+b}{2}\right) \\
& \leq \quad\left[f\left(\frac{(2-t) a+t b}{4}\right)+f\left(\frac{t a+(2-t) b}{4}\right)+\right. \\
&\left.+\frac{1}{h\left(\frac{1}{2}\right)} d\left(\frac{(2-t) a+t b}{4}, \frac{t a+(2-t) b}{4}\right)\right] \\
& \times\left[g\left(\frac{(2-t) a+t b}{4}\right)+g\left(\frac{t a+(2-t) b}{4}\right)+\right. \\
&\left.+\frac{1}{h\left(\frac{1}{2}\right)} d\left(\frac{(2-t) a+t b}{4}, \frac{t a+(2-t) b}{4}\right)\right] \\
&= f\left(\frac{(2-t) a+t b}{4}\right) g\left(\frac{(2-t) a+t b}{4}\right)+
\end{aligned}
$$


164

A. KASHURI, H. BUDAK, R. KAPUCU, AND R. LIKO

$$
\begin{aligned}
& +f\left(\frac{t a+(2-t) b}{4}\right) g\left(\frac{t a+(2-t) b}{4}\right)+ \\
& +f\left(\frac{(2-t) a+t b}{4}\right) g\left(\frac{t a+(2-t) b}{4}\right)+ \\
& +f\left(\frac{t a+(2-t) b}{4}\right) g\left(\frac{(2-t) a+t b}{4}\right) \\
& +\frac{1}{h\left(\frac{1}{2}\right)}\left[f\left(\frac{t a+(2-t) b}{4}\right) d\left(\frac{(2-t) a+t b}{4}, \frac{t a+(2-t) b}{4}\right)\right. \\
& +f\left(\frac{(2-t) a+t b}{4}\right) d\left(\frac{t a+(2-t) b}{4}, \frac{(2-t) a+t b}{4}\right) \\
& +g\left(\frac{t a+(2-t) b}{4}\right) d\left(\frac{(2-t) a+t b}{4}, \frac{t a+(2-t) b}{4}\right) \\
& \left.+g\left(\frac{(2-t) a+t b}{4}\right) d\left(\frac{(2-t) a+t b}{4}, \frac{t a+(2-t) b}{4}\right)\right] \\
& +\frac{1}{h^{2}\left(\frac{1}{2}\right)}\left[d^{2}\left(\frac{(2-t) a+t b}{4}, \frac{t a+(2-t) b}{4}\right)\right] .
\end{aligned}
$$

Multiplying both sides of inequality $(3.28)$ by $\frac{\varphi\left(\left(\frac{b-a}{2}\right) t\right)}{t}$ and integrating the resultant one with respect to $t$ over $[0,1]$, we obtain

$$
\begin{aligned}
& \frac{1}{h^{2}\left(\frac{1}{2}\right)} \int_{0}^{1} \frac{\varphi\left(\left(\frac{b-a}{2}\right) t\right)}{t} f\left(\frac{a+b}{2}\right) g\left(\frac{a+b}{2}\right) d t \\
\leq & \int_{0}^{1} \frac{\varphi\left(\left(\frac{b-a}{2}\right) t\right)}{t} f\left(\frac{(2-t) a+t b}{4}\right) g\left(\frac{(2-t) a+t b}{4}\right) d t \\
+ & \int_{0}^{1} \frac{\varphi\left(\left(\frac{b-a}{2}\right) t\right)}{t} f\left(\frac{t a+(2-t) b}{4}\right) g\left(\frac{t a+(2-t) b}{4}\right) d t \\
+ & \frac{1}{h^{2}\left(\frac{1}{2}\right)} \int_{0}^{1} \frac{\varphi\left(\left(\frac{b-a}{2}\right) t\right)}{t} d^{2}\left(\frac{(2-t) a+t b}{4}, \frac{t a+(2-t) b}{4}\right) d t \\
+ & \frac{1}{h\left(\frac{1}{2}\right)} \int_{0}^{1} \frac{\varphi\left(\left(\frac{b-a}{2}\right) t\right)}{t} f\left(\frac{t a+(2-t) b}{4}\right) d\left(\frac{(2-t) a+t b}{4}, \frac{t a+(2-t) b}{4}\right) d t \\
+ & \frac{1}{h\left(\frac{1}{2}\right)} \int_{0}^{1} \frac{\varphi\left(\left(\frac{b-a}{2}\right) t\right)}{t} f\left(\frac{(2-t) a+t b}{4}\right) d\left(\frac{(2-t) a+t b}{4}, \frac{t a+(2-t) b}{4}\right) d t \\
+ & \frac{1}{h\left(\frac{1}{2}\right)} \int_{0}^{1} \frac{\varphi\left(\left(\frac{b-a}{2}\right) t\right)}{t} g\left(\frac{t a+(2-t) b}{4}\right) d\left(\frac{(2-t) a+t b}{4}, \frac{t a+(2-t) b}{4}\right) d t \\
+ & \frac{1}{h\left(\frac{1}{2}\right)} \int_{0}^{1} \frac{\varphi\left(\left(\frac{b-a}{2}\right) t\right)}{t} g\left(\frac{(2-t) a+t b}{4}\right) d\left(\frac{(2-t) a+t b}{4}, \frac{t a+(2-t) b}{4}\right) d t
\end{aligned}
$$




$$
\begin{aligned}
& +2 M(a, b) \int_{0}^{1} \frac{\varphi\left(\left(\frac{b-a}{2}\right) t\right)}{t} h\left(\frac{t}{2}\right) h\left(\frac{2-t}{2}\right) d t \\
& +N(a, b) \int_{0}^{1} \frac{\varphi\left(\left(\frac{b-a}{2}\right) t\right)}{t}\left[h^{2}\left(\frac{t}{2}\right)+h^{2}\left(\frac{2-t}{2}\right)\right] d t \\
& +P(a, b) d(a, b) \int_{0}^{1} \frac{\varphi\left(\left(\frac{b-a}{2}\right) t\right)}{t}\left[h\left(\frac{t}{2}\right)+h\left(\frac{2-t}{2}\right)\right] d t+ \\
& +2 d^{2}(a, b) \int_{0}^{1} \frac{\varphi\left(\left(\frac{b-a}{2}\right) t\right)}{t} d t .
\end{aligned}
$$

By changing the variable of integration we achieved desired inequality (3.25).

Theorem 9. Let $f:[a, b] \rightarrow \Re$ be an approximately $h$-convex function on $[a, b]$, with $a<b$. Then the following inequalities for generalized fractional integrals hold:

$$
\begin{aligned}
& \frac{1}{2 h\left(\frac{1}{2}\right)} f\left(\frac{a+b}{2}\right)-A_{3} \\
\leq & \frac{1}{2 \Psi(1)}\left[a+I_{\varphi} f\left(\frac{a+b}{2}\right)+{ }_{b-} I_{\varphi} f\left(\frac{a+b}{2}\right)\right] \\
\leq & \frac{[f(a)+f(b)]}{2 \Psi(1)} \int_{0}^{1} \frac{\varphi\left(\left(\frac{b-a}{2}\right) t\right)}{t}\left[h\left(\frac{1-t}{2}\right)+h\left(\frac{1+t}{2}\right)\right] d t+d(a, b),
\end{aligned}
$$

where

$$
A_{3}:=\frac{1}{2 \Psi(1) h\left(\frac{1}{2}\right)} \int_{\frac{a+b}{2}}^{b} \frac{\varphi\left(x-\frac{a+b}{2}\right)}{x-\frac{a+b}{2}} d(x, a+b-x) d x .
$$

Proof. Since $f$ is approximately $h$-convex, we have the following inequality

$$
f\left(\frac{x+y}{2}\right) \leq h\left(\frac{1}{2}\right)[f(x)+f(y)]+d(x, y) .
$$

For $x=\frac{(1-t)}{2} a+\frac{(1+t)}{2} b$ and $y=\frac{(1-t)}{2} b+\frac{(1+t)}{2} a$ with $t \in[0,1], 3.31$ becomes

$$
\begin{aligned}
\frac{1}{h\left(\frac{1}{2}\right)} f\left(\frac{a+b}{2}\right) \leq & f\left(\frac{(1-t)}{2} a+\frac{(1+t)}{2} b\right) \\
& +f\left(\frac{(1-t)}{2} b+\frac{(1+t)}{2} a\right) \\
& +\frac{1}{h\left(\frac{1}{2}\right)} d\left(\frac{(1-t)}{2} a+\frac{(1+t)}{2} b, \frac{(1-t)}{2} b+\frac{(1+t)}{2} a\right) .
\end{aligned}
$$

Multiplying both sides of the inequality 3.32 by $\frac{\varphi\left(\left(\frac{b-a}{2}\right) t\right)}{t}$ and integrating the resultant inequality with respect to $t$ over $[0,1]$, we have

$$
\frac{1}{h\left(\frac{1}{2}\right)} f\left(\frac{a+b}{2}\right) \int_{0}^{1} \frac{\varphi\left(\left(\frac{b-a}{2}\right) t\right)}{t} d t
$$




$$
\begin{aligned}
\leq & \int_{0}^{1} \frac{\varphi\left(\left(\frac{b-a}{2}\right) t\right)}{t} f\left(\frac{(1-t)}{2} a+\frac{(1+t)}{2} b\right) d t+ \\
& +\int_{0}^{1} \frac{\varphi\left(\left(\frac{b-a}{2}\right) t\right)}{t} f\left(\frac{(1-t)}{2} b+\frac{(1+t)}{2} a\right) d t \\
& +\frac{1}{h\left(\frac{1}{2}\right)} \int_{0}^{1} \frac{\varphi\left(\left(\frac{b-a}{2}\right) t\right)}{t} d\left(\frac{(1-t)}{2} a+\frac{(1+t)}{2} b, \frac{(1-t)}{2} b+\frac{(1+t)}{2} a\right) d t .
\end{aligned}
$$

As a consequence, we get

$$
\begin{aligned}
\frac{1}{2 h\left(\frac{1}{2}\right)} f\left(\frac{a+b}{2}\right) \leq & \frac{1}{2 \Psi(1)}\left[{ }_{a+} I_{\varphi} f\left(\frac{a+b}{2}\right)+{ }_{b-} I_{\varphi} f\left(\frac{a+b}{2}\right)\right] \\
& +\frac{1}{2 \Psi(1) h\left(\frac{1}{2}\right)} \int_{\frac{a+b}{2}}^{b} \frac{\varphi\left(x-\frac{a+b}{2}\right)}{x-\frac{a+b}{2}} d(x, a+b-x) d x
\end{aligned}
$$

and thus the first inequality is proved.

To obtain the second inequality in $(3.30)$, since $f$ is an approximately $h$-convex, we have

$$
\begin{aligned}
& f\left(\frac{(1-t)}{2} a+\frac{(1+t)}{2} b\right)+f\left(\frac{(1-t)}{2} b+\frac{(1+t)}{2} a\right) \\
\leq & {[f(a)+f(b)]\left[h\left(\frac{1-t}{2}\right)+h\left(\frac{1+t}{2}\right)\right]+2 d(a, b) . }
\end{aligned}
$$

Then multiplying both sides of 3.33 by $\frac{\varphi\left(\left(\frac{b-a}{2}\right) t\right)}{t}$ and integrating the resultant inequality with respect to $t$ on $[0,1]$, we get

$$
\begin{aligned}
& \int_{0}^{1} \frac{\varphi\left(\left(\frac{b-a}{2}\right) t\right)}{t} f\left(\frac{(1-t)}{2} a+\frac{(1+t)}{2} b\right) d t \\
& +\int_{0}^{1} \frac{\varphi\left(\left(\frac{b-a}{2}\right) t\right)}{t} f\left(\frac{(1-t)}{2} b+\frac{(1+t)}{2} a\right) d t \\
& \leq \quad[f(a)+f(b)] \int_{0}^{1} \frac{\varphi\left(\left(\frac{b-a}{2}\right) t\right)}{t}\left[h\left(\frac{(1-t)}{2}\right)+h\left(\frac{1+t}{2}\right)\right] d t+2 d(a, b) \Psi(1) .
\end{aligned}
$$

As a sequel, we obtain

$$
\frac{1}{2 \Psi(1)}\left[{ }_{a+} I_{\varphi} f\left(\frac{a+b}{2}\right)+{ }_{b-} I_{\varphi} f\left(\frac{a+b}{2}\right)\right]
$$




$$
\leq \frac{[f(a)+f(b)]}{2 \Psi(1)} \int_{0}^{1} \frac{\varphi\left(\left(\frac{b-a}{2}\right) t\right)}{t}\left[h\left(\frac{(1-t)}{2}\right)+h\left(\frac{1+t}{2}\right)\right] d t+d(a, b)
$$

and the second inequality is proved. The proof is completed.

Theorem 10. Let $f, g:[a, b] \rightarrow \Re$ are two approximately $h$-convex functions on $[a, b]$, with $a<b$. Then the following inequality for generalized fractional integrals holds:

$$
\begin{aligned}
& \frac{1}{2}\left[{ }_{b-} I_{\varphi} f\left(\frac{a+b}{2}\right) g\left(\frac{a+b}{2}\right)+{ }_{a+} I_{\varphi} f\left(\frac{a+b}{2}\right) g\left(\frac{a+b}{2}\right)\right] \\
\leq & M_{1} M(a, b)+M_{2} N(a, b)+M_{3} P(a, b) d(a, b)+d^{2}(a, b) \Psi(1),
\end{aligned}
$$

where $M(a, b), N(a, b)$ and $P(a, b)$ are defined as in Theorem 4 and

$$
\begin{aligned}
& M_{1}:=\frac{1}{2} \int_{0}^{1} \frac{\varphi\left(\frac{(b-a)}{2} t\right)}{t}\left[h^{2}\left(\frac{1-t}{2}\right)+h^{2}\left(\frac{1+t}{2}\right)\right] d t, \\
& M_{2}:=\int_{0}^{1} \frac{\varphi\left(\frac{(b-a)}{2} t\right)}{t}\left[h\left(\frac{1-t}{2}\right) h\left(\frac{1+t}{2}\right)\right] d t, \\
& M_{3}:=\frac{1}{2} \int_{0}^{1} \frac{\varphi\left(\frac{(b-a)}{2} t\right)}{t}\left[h\left(\frac{1-t}{2}\right)+h\left(\frac{1+t}{2}\right)\right] d t .
\end{aligned}
$$

Proof. Since $f$ and $g$ are two approximately $h$-convex functions for $t \in[0,1]$, we have

$$
f\left(\frac{(1-t) a+(1+t) b}{2}\right) \leq h\left(\frac{1-t}{2}\right) f(a)+h\left(\frac{1+t}{2}\right) f(b)+d(a, b)
$$

and

$$
g\left(\frac{(1-t) a+(1+t) b}{2}\right) \leq h\left(\frac{1-t}{2}\right) g(a)+h\left(\frac{1+t}{2}\right) g(b)+d(a, b)
$$

Multiplying (3.34) and (3.35), we get

$$
\begin{aligned}
& f\left(\frac{(1-t) a+(1+t) b}{2}\right) g\left(\frac{(1-t) a+(1+t) b}{2}\right) \\
\leq & h^{2}\left(\frac{1-t}{2}\right) f(a) g(a)+h^{2}\left(\frac{1+t}{2}\right) f(b) g(b) \\
& +h\left(\frac{1-t}{2}\right) h\left(\frac{1+t}{2}\right)[f(a) g(b)+f(b) g(a)] \\
& +h\left(\frac{1-t}{2}\right) d(a, b)[f(a)+g(a)]+h\left(\frac{1+t}{2}\right) d(a, b)[f(b)+g(b)]+d^{2}(a, b) .
\end{aligned}
$$


Similarly, we obtain

$$
\begin{aligned}
& f\left(\frac{(1+t) a+(1-t) b}{2}\right) g\left(\frac{(1+t) a+(1-t) b}{2}\right) \\
\leq & h^{2}\left(\frac{1-t}{2}\right) f(b) g(b)+h^{2}\left(\frac{1+t}{2}\right) f(a) g(a) \\
& +h\left(\frac{1-t}{2}\right) h\left(\frac{1+t}{2}\right)[f(b) g(a)+f(a) g(b)] \\
& h\left(\frac{1-t}{2}\right) d(a, b)[f(b)+g(b)]+h\left(\frac{1+t}{2}\right) d(a, b)[f(a)+g(a)]+d^{2}(a, b) .
\end{aligned}
$$

Adding 3.36 and (3.37), we have following relation

$$
\begin{aligned}
& f\left(\frac{(1-t) a+(1+t) b}{2}\right) g\left(\frac{(1-t) a+(1+t) b}{2}\right) \\
& +f\left(\frac{(1+t) a+(1-t) b}{2}\right) g\left(\frac{(1+t) a+(1-t) b}{2}\right) \\
\leq \quad & {\left[h^{2}\left(\frac{1-t}{2}\right)+h^{2}\left(\frac{1+t}{2}\right)\right] M(a, b)+2 h\left(\frac{1-t}{2}\right) h\left(\frac{1+t}{2}\right) N(a, b) } \\
& {\left[h\left(\frac{1-t}{2}\right)+h\left(\frac{1+t}{2}\right)\right] d(a, b) P(a, b)+2 d^{2}(a, b) . }
\end{aligned}
$$

Multiplying both sides of 3.38 by $\frac{\varphi\left(\frac{(b-a)}{2} t\right)}{t}$ and integrating the resultant one with respect to t over $[0,1]$, we get

$$
\begin{aligned}
& \int_{0}^{1} \frac{\varphi\left(\frac{b-a}{2} t\right)}{t} f\left(\frac{(1-t) a+(1+t) b}{2}\right) g\left(\frac{(1-t) a+(1+t) b}{2}\right) d t \\
& +\int_{0}^{1} \frac{\varphi\left(\frac{b-a}{2} t\right)}{t} f\left(\frac{(1+t) a+(1-t) b}{2}\right) g\left(\frac{(1+t) a+(1-t) b}{2}\right) d t \\
& \leq \quad M(a, b) \int_{0}^{1} \frac{\varphi\left(\frac{b-a}{2} t\right)}{t}\left[h^{2}\left(\frac{1-t}{2}\right)+h^{2}\left(\frac{1+t}{2}\right)\right] d t \\
& +2 N(a, b) \int_{0}^{1} \frac{\varphi\left(\frac{b-a}{2} t\right)}{t} h^{2}\left(\frac{1-t}{2}\right) h^{2}\left(\frac{1+t}{2}\right) d t \\
& +P(a, b) d(a, b) \int_{0}^{1} \frac{\varphi\left(\frac{b-a}{2} t\right)}{t}\left[h\left(\frac{1-t}{2}\right)+h\left(\frac{1+t}{2}\right)\right] d t
\end{aligned}
$$




$$
+d^{2}(a, b) \int_{0}^{1} \frac{\varphi\left(\frac{b-a}{2} t\right)}{t} d t .
$$

This completes the proof.

Theorem 11. Let $f, g:[a, b] \rightarrow \Re$ are two approximately $h$-convex functions on $[a, b]$, with $a<b$. Then the following inequality for generalized fractional integrals holds:

$$
\begin{aligned}
& \quad \frac{1}{2 h^{2}\left(\frac{1}{2}\right)} f\left(\frac{a+b}{2}\right) g\left(\frac{a+b}{2}\right) \\
& -\frac{1}{2 \Psi(1) h\left(\frac{1}{2}\right)}\left[\int_{\frac{a+b}{2}}^{b} \frac{\varphi\left(x-\frac{a+b}{2}\right)}{x-\frac{a+b}{2}}[f(x)+g(x)] d(x, a+b-x) d x\right. \\
& \left.\quad+\int_{\frac{a+b}{2}}^{b} \frac{\varphi\left(\frac{a+b}{2}-x\right)}{\frac{a+b}{2}-x}[f(x)+g(x)] d(a+b-x, x) d x\right] \\
& \leq \quad \frac{1}{2}\left[b-I_{\varphi} f\left(\frac{a+b}{2}\right) g\left(\frac{a+b}{2}\right)+{ }_{a+} I_{\varphi} f\left(\frac{a+b}{2}\right) g\left(\frac{a+b}{2}\right)\right] \\
& \quad+\frac{1}{2 \Psi(1) h^{2}\left(\frac{1}{2}\right)} \int_{\frac{a+b}{2}}^{b} \frac{\varphi\left(x-\frac{a+b}{2}\right)}{x-\frac{a+b}{2}} d(x, a+b-x) d x \\
& \quad+\frac{1}{\Psi(1)}\left[M_{2} M(a, b)+M_{1} N(a, b)+M_{3} P(a, b) d(a, b)\right]+d^{2}(a, b),
\end{aligned}
$$

where $M(a, b), N(a, b), P(a, b)$ and $M_{1}, M_{2}, M_{3}$ are defined as in Theorems 4 and 10 , respectively.

Proof. For $t \in[0,1]$, we can write

$$
\frac{a+b}{2}=\frac{(1-t) a+(1+t) b}{4}+\frac{(1+t) a+(1-t) b}{4} .
$$

Since $f$ and $g$ are two approximately $h$-convex functions, we have

$$
\begin{gathered}
\frac{1}{h\left(\frac{1}{2}\right)} f\left(\frac{a+b}{2}\right)= \\
=\frac{1}{h\left(\frac{1}{2}\right)} \times f\left(\frac{(1-t) a+(1+t) b}{4}+\frac{(1+t) a+(1-t) b}{4}\right) \\
\leq f\left(\frac{(1-t) a+(1+t) b}{4}\right)+f\left(\frac{(1+t) a+(1-t) b}{4}\right) \\
+\frac{1}{h\left(\frac{1}{2}\right)} d\left(\frac{(1-t) a+(1+t) b}{4}, \frac{(1+t) a+(1-t) b}{4}\right)
\end{gathered}
$$


and similarly

$$
\begin{aligned}
\frac{1}{h\left(\frac{1}{2}\right)} g\left(\frac{a+b}{2}\right) & \leq g\left(\frac{(1-t) a+(1+t) b}{4}\right) \\
& +g\left(\frac{(1+t) a+(1-t) b}{4}\right) \\
& +\frac{1}{h\left(\frac{1}{2}\right)} d\left(\frac{(1-t) a+(1+t) b}{4}, \frac{(1+t) a+(1-t) b}{4}\right) .
\end{aligned}
$$

Multiplying the inequalities 3.40 and 3.41 , we obtain

$$
\begin{aligned}
& \frac{1}{h^{2}\left(\frac{1}{2}\right)} f\left(\frac{a+b}{2}\right) g\left(\frac{a+b}{2}\right) \\
& \leq\left[f\left(\frac{(1-t) a+(1+t) b}{4}\right)+f\left(\frac{(1+t) a+(1-t) b}{4}\right)\right. \\
& \left.+\frac{1}{h\left(\frac{1}{2}\right)} d\left(\frac{(1-t) a+(1+t) b}{4}, \frac{(1+t) a+(1-t) b}{4}\right)\right] \\
& \times\left[g\left(\frac{(1-t) a+(1+t) b}{4}\right)+g\left(\frac{(1+t) a+(1-t) b}{4}\right)\right. \\
& \left.+\frac{1}{h\left(\frac{1}{2}\right)} d\left(\frac{(1-t) a+(1+t) b}{4}, \frac{(1+t) a+(1-t) b}{4}\right)\right] \\
& =f\left(\frac{(1-t) a+(1+t) b}{4}\right) g\left(\frac{(1-t) a+(1+t) b}{4}\right) \\
& +f\left(\frac{(1+t) a+(1-t) b}{4}\right) g\left(\frac{(1+t) a+(1-t) b}{4}\right) \\
& +\left[f\left(\frac{(1-t) a+(1+t) b}{4}\right) g\left(\frac{(1+t) a+(1-t) b}{4}\right)\right. \\
& \left.+f\left(\frac{(1+t) a+(1-t) b}{4}\right) g\left(\frac{(1-t) a+(1+t) b}{4}\right)\right] \\
& +\frac{1}{h\left(\frac{1}{2}\right)}\left[f\left(\frac{(1-t) a+(1+t) b}{4}\right)\right. \\
& d\left(\frac{(1-t) a+(1+t) b}{4}, \frac{(1+t) a+(1-t) b}{4}\right) \\
& +f\left(\frac{(1+t) a+(1-t) b}{4}\right) d\left(\frac{(1-t) a+(1+t) b}{4}, \frac{(1+t) a+(1-t) b}{4}\right) \\
& +g\left(\frac{(1-t) a+(1+t) b}{4}\right) d\left(\frac{(1-t) a+(1+t) b}{4}, \frac{(1+t) a+(1-t) b}{4}\right)
\end{aligned}
$$


NEW INEQUALITIES FOR APPROXIMATELY $h$-CONVEX FUNCTIONS

$$
\begin{aligned}
& \left.+g\left(\frac{(1+t) a+(1-t) b}{4}\right) d\left(\frac{(1-t) a+(1+t) b}{4}, \frac{(1+t) a+(1-t) b}{4}\right)\right] \\
& +\frac{1}{h^{2}\left(\frac{1}{2}\right)}\left[d^{2}\left(\frac{(1-t) a+(1+t) b}{4}, \frac{(1+t) a+(1-t) b}{4}\right)\right] .
\end{aligned}
$$

By using the approximately $h$-convexity of $f$ and $g$, we get

$$
\begin{aligned}
& \frac{1}{h^{2}\left(\frac{1}{2}\right)} f\left(\frac{a+b}{2}\right) g\left(\frac{a+b}{2}\right) \\
\leq & f\left(\frac{(1-t) a+(1+t) b}{4}\right) g\left(\frac{(1-t) a+(1+t) b}{4}\right) \\
& +f\left(\frac{(1+t) a+(1-t) b}{4}\right) g\left(\frac{(1+t) a+(1-t) b}{4}\right) \\
& +N(a, b)\left[h^{2}\left(\frac{1-t}{2}\right)+h^{2}\left(\frac{1+t}{2}\right)\right] \\
& +2 M(a, b) h\left(\frac{1-t}{2}\right) h\left(\frac{1+t}{2}\right) \\
& +d(a, b) P(a, b)\left[h\left(\frac{1-t}{2}\right)+h\left(\frac{1+t}{2}\right)\right]+2 d^{2}(a, b) \\
& +\frac{1}{h\left(\frac{1}{2}\right)}\left[f\left(\frac{(1-t) a+(1+t) b}{4}\right)\right. \\
& +f\left(\frac{(1+t) a+(1-t) b}{4}\right) d\left(\frac{(1-t) a+(1+t) b}{4}, \frac{(1+t) a+(1-t) b}{4}\right) \\
+ & g\left(\frac{(1-t) a+(1+t) b}{4}\right) d\left(\frac{(1-t) a+(1+t) b}{4}, \frac{(1+t) a+(1-t) b}{4}\right) \\
+ & \left.g\left(\frac{(1+t) a+(1-t) b}{4}\right) d\left(\frac{(1-t) a+(1+t) b}{4}, \frac{(1+t) a+(1-t) b}{4}\right)\right] \\
+ & \frac{1}{h^{2}\left(\frac{1}{2}\right)} d^{2}\left(\frac{(1-t) a+(1+t) b}{4}, \frac{(1+t) a+(1-t) b}{4}\right) .
\end{aligned}
$$

Multiplying both sides of 3.43 by $\frac{\varphi\left(\frac{(b-a)}{2} t\right)}{t}$ and integrating the resultant one with respect to $t$ over $[0,1]$, we get

$$
\frac{1}{h^{2}\left(\frac{1}{2}\right)} \int_{0}^{1} \frac{\varphi\left(\frac{b-a}{2} t\right)}{t} f\left(\frac{a+b}{2}\right) g\left(\frac{a+b}{2}\right) d t
$$




$$
\begin{aligned}
& \leq \int_{0}^{1} \frac{\varphi\left(\frac{b-a}{2} t\right)}{t} f\left(\frac{(1-t) a+(1+t) b}{4}\right) g\left(\frac{(1-t) a+(1+t) b}{4}\right) d t \\
& +\int_{0}^{1} \frac{\varphi\left(\frac{b-a}{2} t\right)}{t} f\left(\frac{(1+t) a+(1-t) b}{4}\right) g\left(\frac{(1+t) a+(1-t) b}{4}\right) d t \\
& +N(a, b) \int_{0}^{1} \frac{\varphi\left(\frac{b-a}{2} t\right)}{t}\left[h^{2}\left(\frac{1-t}{2}\right)+h^{2}\left(\frac{1+t}{2}\right)\right] d t \\
& +2 M(a, b) \int_{0}^{1} \frac{\varphi\left(\frac{b-a}{2} t\right)}{t} h\left(\frac{1-t}{2}\right) h\left(\frac{1+t}{2}\right) d t \\
& +P(a, b) d(a, b) \int_{0}^{1} \frac{\varphi\left(\frac{b-a}{2} t\right)}{t}\left[h\left(\frac{1-t}{2}\right)+h\left(\frac{1+t}{2}\right)\right] d t \\
& +2 d^{2}(a, b) \int_{0}^{1} \frac{\varphi\left(\frac{b-a}{2} t\right)}{t} d t \\
& +\int_{0}^{1} \frac{\varphi\left(\frac{b-a}{2} t\right)}{t} g\left(\frac{(1+t) a+(1-t) b}{4}\right) \\
& +\frac{1}{h\left(\frac{1}{2}\right)}\left[\int_{0}^{1} \frac{\varphi\left(\frac{b-a}{2} t\right)}{t} f\left(\frac{(1-t) a+(1+t) b}{4}\right)\right. \\
& +\int_{0}^{1} \frac{\varphi\left(\frac{b-a}{2} t\right)}{t} f\left(\frac{(1+t) a+(1-t) b}{4}\right) \\
& \left.+\frac{(1-t) a+(1+t) b}{4}, \frac{(1+t) a+(1-t) b}{4}\right) d t \\
& + \\
& \left.+\frac{(1-t) a+(1+t) b}{4}, \frac{(1+t) a+(1-t) b}{4}\right) d t
\end{aligned}
$$




$$
+\frac{1}{h^{2}\left(\frac{1}{2}\right)} \int_{0}^{1} \frac{\varphi\left(\frac{b-a}{2} t\right)}{t} d^{2}\left(\frac{(1-t) a+(1+t) b}{4}, \frac{(1+t) a+(1-t) b}{4}\right) d t .
$$

By changing the variable of integration we achieved desired inequality (3.39).

Remark 3.1: Applying the above results for suitable options of function $\varphi(t)=$ $t, \frac{t^{\alpha}}{\Gamma(\alpha)}, \frac{t^{\frac{\alpha}{k}}}{k \Gamma_{k}(\alpha)} ; \varphi(t)=t(b-t)^{\alpha-1}$ for $\alpha \in(0,1) ; \varphi(t)=\frac{t}{\alpha} \exp (-A t)$, where $A=\frac{1-\alpha}{\alpha}$ for $\alpha \in(0,1)$, and if we take, respectively, $h(t)=1, t, t^{s}, \frac{1}{t^{s}}, t(1-t)$, and $h(t)=\frac{\sqrt{1-t}}{2 \sqrt{t}}$, such that $f$ to be approximately $h$-convex function, we can construct some new generalized fractional integral inequalities. We omit their proofs and the details are left to the interested readers.

\section{Conclusion}

Since the functions which are approximately $h$-convex have large applications in many mathematical areas, our results can be applied to obtain several new fascinating inequalities in convex analysis, special functions, quantum mechanics, related optimization theory, mathematical inequalities. Also, we can obtain several new general fractional integral inequalities using special means (arithmetic, geometric, logarithmic, etc.). Finally, some new bounds for the midpoint and trapezium quadrature formula using our results can be provided as well. Our results may stimulate further research in different areas of pure and applied sciences.

Acknowledgements. The authors would like to thank the honorable referees and editors for valuable comments and suggestions for improved our manuscript.

\section{REFERENCES}

[1] W. W. Breckner, Stetigkeitsaussagen für eine Klasse verallgemeinerter konvexer funktionen in topologischen linearen Räumen, Publ. Inst. Math., 23 (1978), 13-20.

[2] H. Budak, M. Z. Sarikaya, M. K. Yildiz, Hermite-Hadamard type inequalities for F-convex function involving fractional integrals, Filomat, 32(16) (2018), 5509-5518.

[3] H. Budak, F. Ertuğral, M. Z. Sarikaya, New generalization of Hermite-Hadamard type inequalities via generalized fractional integrals, (2018), Submitted.

[4] P. Burai, A. Házy, On approximately h-convex functions, J. Convex Anal., 18(2) (2011), $447-454$.

[5] H. Chen, U. N. Katugampola, Hermite-Hadamard and Hermite-Hadamard-Fejér type inequalities for generalized fractional integrals, J. Math. Anal. Appl., 446 (2017), 1274-1291.

[6] S. S. Dragomir, C. E. M. Pearce, Selected Topics on Hermite-Hadamard Inequalities and Applications, RGMIA Monographs, Victoria University, 2000.

[7] S. S. Dragomir, R. P. Agarwal, Two inequalities for differentiable mappings and applications to special means of real numbers and to trapezoidal formula, Appl. Math. Lett., 11(5) (1998), $91-95$.

[8] S. S. Dragomir, S. Fitzpatrik, The Hadamard's inequality for s-convex functions in the second sense, Demonstratio Math., 32(4) (1999), 687-696. 
[9] G. Farid, A. Rehman, M. Zahra, On Hadamard type inequalities for $k$-fractional integrals, Konurap J. Math., 4(2) (2016), 79-86.

[10] G. Farid, A. Rehman, M. Zahra, On Hadamard inequalities for $k$-fractional integrals, Nonlinear Funct. Anal. Appl., 21(3) (2016), 463-478.

[11] R. Gorenflo, F. Mainardi, Fractional calculus: integral and differential equations of fractional order, Springer Verlag, Wien, (1997), 223-276.

[12] J. Hadamard, Etude sur les proprietes des fonctions entieres en particulier d'une fonction consideree par Riemann, J. Math. Pures Appl., 58 (1893), 171-215.

[13] R. Hussain, A. Ali, A. Latif, G. Gulshan, Some k-fractional associates of HermiteHadamard's inequality for quasi-convex functions and applications to special means, Fract. Differ. Calc., 7(2) (2017), 301-309.

[14] M. Iqbal, S. Qaisar, M. Muddassar, A short note on integral inequality of type HermiteHadamard through convexity, J. Comput. Anal. Appl., 21(5) (2016), 946-953.

[15] İ. İşcan, S. Wu, Hermite-Hadamard type inequalities for harmonically convex functions via fractional integrals, Appl. Math. Compt., 238 (2014), 237-244.

[16] İ. İşcan, On generalization of different type integral inequalities for $s$-convex functions via fractional integrals, Math. Sci. Appl., 2(1) (2014), 55-67.

[17] M. Jleli, B. Samet On Hermite-Hadamard type inequalities via fractional integrals of a function with respect to another function, J. Nonlinear Sci. Appl., 9(3) (2016), 1252-1260.

[18] U. N. Katugampola, New approach to a generalized fractional integral, Appl. Math. Comput., 218(3) (2011), 860-865.

[19] R. Khalil, M. Al Horani, A. Yousef, M. Sababheh, A new definition of fractional derivative, J. Comput. Appl. Math., 264 (2017), 65-70.

[20] A. A. Kilbas, H. M. Srivastava, J. J. Trujillo, Theory and Applications of Fractional Differential Equations, North-Holland Mathematics Studies, 204, Elsevier Sci. B.V., Amsterdam, 2006.

[21] U. S. Kırmac1, Inequalities for differentiable mappings and applications to special means of real numbers to midpoint formula, Appl. Math. Comput., 147(5) (2004), 137-146.

[22] U. S. Kırmacı, M. K. Bakula, M. E. Özdemir, J. Pečarić, Hadamard-type inequalities for s-convex functions, Appl. Math. Comput., 193 (2007), 26-35.

[23] S. Mubeen, G. M Habibullah, k-Fractional integrals and application, Int. J. Contemp. Math. Sciences, 7(2) (2012), 89-94.

[24] M. A. Noor, M. U. Awan, Some integral inequalities for two kinds of convexities via fractional integrals, TJMM, 5(2) (2013), 129-136.

[25] M. E. Özdemir, M. A. Ardıç, H. Kavurmacı, Hermite-Hadamard type inequalities for sconvex and s-concave functions via fractional integrals, Turkish J. Science, 1(1) (2016), $28-40$.

[26] M. E. Özdemir, H. Kavurmaci, Yildiz, Fractional integral inequalities via s-convex functions Turkish Journal of Analysis and Number Theory, 5(1) (2017), 18-22.

[27] M. E. Özdemir, S. S. Dragomir, Ç. Yildiz, The Hadamard inequality for convex functions via fractional integrals, Acta Math. Sci., Ser. A, Chin. Ed., 33A(5) (2013), 1293-1299.

[28] J. E. Pečarić, F. Proschan, Y. L. Tong, Convex Functions, Partial Orderings and Statistical Applications, Academic Press, Boston, 1992.

[29] C. E. M. Pearce, J. Pečarić, Inequalities for Differentiable Mappings with Application to Special Means and Quadrature Formula, Appl. Math. Lett., 13 (2000), 51-55.

[30] A. Saglam, M. Z. Sarikaya, H. Yildirim, Some new inequalities of Hermite-Hadamard's type, Kyungpook Math. J., 50 (2010), 399-410.

[31] M. Z. Sarikaya, F. Ertuğral, On the generalized Hermite-Hadamard inequalities, Ann. Univ. Craiova Math. Comput. Sci. Ser. 47 (2020), 193-213.

[32] M. Z. Sarikaya, H. Yildirim, On Hermite-Hadamard type inequalities for Riemann-Liouville fractional integrals, Miskolc Math. Notes, 7(2) (2016), 1049-1059.

[33] M. Z. Sarikaya, E. Set, H. Yaldiz, N. Basak, Hermite-Hadamard's inequalities for fractional integrals and related fractional inequalities, Math. Comput. Modelling, 57 (2013) 2403-2407. 
[34] M. Z. Sarikaya, H. Budak, Generalized Hermite-Hadamard type integral inequalities for fractional integrals, Filomat, 30(5) (2016), 1315-1326.

[35] M. Z. Sarikaya, A. Saglam, H. Yildirim, On some Hadamard-type inequalities for $h$-convex functions. J. Math. Inequal., 2(3) (2008), 335-341.

[36] E. Set, M. Z. Sarikaya, M. E. Ozdemir, H. Yildirim, The Hermite-Hadamard's inequality for some convex functions via fractional integrals and related results, J. Appl. Math. Stat. Inform., 10(2) (2014).

[37] E. Set, M. Z. Sarikaya, M. E. Ozdemir, H. Yildirim,The Hadamard's inequality for s-convex functions in the second sense via fractional integrals and related results, Submitted.

[38] M. Tunç, On new inequalities for $h$-convex functions via Riemann-Liouville fractional integration, Filomat, 27(4) (2013), 559-565.

[39] J. Wang, X. Li, M. Fečkan, Y. Zhou, Hermite-Hadamard-type inequalities for RiemannLiouville fractional integrals via two kinds of convexity, Appl. Anal., 92(11) (2012), 22412253.

[40] J. Wang, X. Li, C. Zhu, Refinements of Hermite-Hadamard type inequalities involving fractional integrals, Bull. Belg. Math. Soc. Simon Stevin, 20 (2013), 655-666.

[41] S. Varošanec, On h-convexity, J. Math. Anal. Appl., 326 (2007), 303-311.

[42] Y. Zhang, J. Wang, On some new Hermite-Hadamard inequalities involving RiemannLiouville fractional integrals, J. Inequal. Appl., 2013(220) (2013).

[43] A. Kashuri, R. Liko, Some new Hermite-Hadamard type inequalities and their applications, Stud. Sci. Math. Hung., 56(1) (2019), 103-142.

[44] P. O. Mohammed, T. Abdeljawad, S. Zeng, A. Kashuri, Fractional Hermite-Hadamard integral inequalities for a new class of convex functions, Symmetry, 12 (2020), 1485.

[45] D. Baleanu, A. Kashuri, P. O. Mohammed, B. Meftah, General Raina fractional integral inequalities on coordinates of convex functions, Adv. Difference Equ., 2021 (2021), 82.

\footnotetext{
1 Department of Mathematics, Faculty of Technical Science, University "Ismail Qemali", 9400 Vlora, Albania

2 Department of Mathematics, Faculty of Science and Arts, Düzce University,

DÜZCE-TURKEY

Email address: hsyn.budak@gmail.com

Email address: rabiakapucu.7@gmail.com
}

Received 2.9.2021

Revised 13.1.2022

Accepted 17.1.2022 